\title{
Effects of Collisions on Whistler-Mode Ray Tracing
}

\author{
Kunihiro SuzukI and Kiyohide BABA \\ Chubu Institute of Technology, Kasugai, Japan
}

(Received May 11, 1972; Revised January 23, 1973)

\begin{abstract}
The collisional effects on ray tracing are considered. Ray equations suitable for inhomogeneous waves are derived and some whistler ray paths are numerically computed in the case of penetration through the bottom of the ionosphere.
\end{abstract}

\section{Introduction}

A set of differential equations for ray tracing suitable for inhomogeneous, anisotropic medium has been established by some researchers (BUDDEN, 1961; HASELGROVE, 1955). In the last decade, many calculations of whistler ray paths have been made in order to investigate how the ray paths vary with frequency, initial latitude, initial wave normal angle, electron density profile and effect of ions (YABROFF, 1961; KimURA, 1966). These works may be sufficient for a basis to understand the experimental data of whistler phenomena, but details on the effects of collision on ray tracing should be added to these calculations.

It is the main purpose of this paper to extend the ray equation for the purpose of including collisional effects. The so-called inhomogeneous wave must be treated, i.e., the direction of its wave normal is expressed by the complex angle. The reason a wave of this kind must be treated, results from the fact that Snell's law is satisfied, and the refractive index is a complex vector because of finite collisional effects. This wave is what the Booker quartic equation involves, and the direction of the phase propagation of this wave is different from the direction in which the wave amplitude changes most rapidly. This fact is overlooked in the ray equation involving collisional terms in the reference (YABrofF, 1961). Many discussions have been made by Hines (1951) as to the ray direction of the inhomogeneous wave. We can calculate the ray paths, including the collisional effects, by using the result by Hines (1951) together with the canonical equation for a ray path (Budden, 1961; Haselgrove, 1955).

The next problem is to apply our method to the actual condition. As Kimura (1966) points out, collisional effects are ineffective except in the narrow region near the bottom of the ionosphere. The neglect of collisional effects on ray tracing is reasonable for the purpose of the investigation of the macroscopic characteristics such as the value of the dispersion of whistler waves observed. In order to find the collisional effects, we must consider the behavior of whistler 
waves at this lower boundary of the ionosphere, where the effects of the collisions between electrons and neutral particles are predominant. The ray-treatment of such a low frequency wave in this narrow region is not suitable; this will be discussed in the last section. However, we show our numerical results in order to have a vision of the propagating whistler waves, and to show how to penetrate the bottom of the ionosphere and propagate into free space and from which direction the whistlers come to our receiver, until this question is solved calculating the wave equation exactly in the inhomogeneous medium. Our problem was already treated by MAEDA and OyA (1963). They showed the ray direction of VLF radio waves penetrating through the ionosphere by finding the solution to the wave equation in the form of the plane wave, which satisfies the boundary condition at the sharp boundary between the free space and the ionosphere (with higher electron density than ours). We will discuss the application of their procedure to find the ray direction, which was based on the direction of the Poynting-vector, to the consideration of the collisional effects in the last section.

We take a model of the flat ionosphere which is appropriate to describe the local and narrow region at the bottom of the ionosphere. Our treatment is restricted to waves in the plane of the magnetic meridian, i.e., the two dimensional case.

\section{Ray Tracing of Inhomogeneous Waves}

Considering a straight sharp boundary between homogeneous plasma and free space, the refractive index vector $\boldsymbol{M}$ of the inhomogeneous wave being coupled with the plane wave in free space can be written as

$$
M=\mu-i \chi,
$$

in the plasma where $i=\sqrt{-1}$. The boundary is expressed as the $x$ axis as shown in Fig. 1, and vector $\chi$ moves toward the $z$ direction by Snell's law. We note

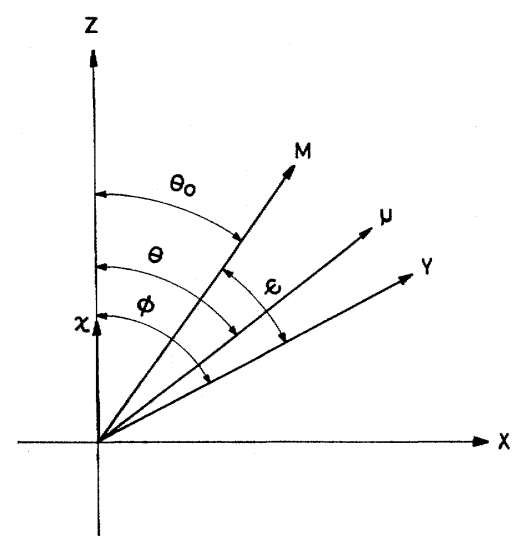

Fig. 1. Vectors, $\mu, \boldsymbol{\chi}, \boldsymbol{M}$ and $\boldsymbol{Y}$ are shown in our coordinate system. 
that the direction of a real wave normal vector $\mu$ is different from the direction of vector $\chi$ in which the amplitude of the wave changes very rapidly. Consequently, the angle between $z$ direction and $\boldsymbol{M}, \theta_{0}$, and the angle between $\boldsymbol{M}$ and $\boldsymbol{Y}$ (magnetic field vector), $\phi$, are complex.

Now, we express the ray path by use of the propagation of the wave packet as follows:

$$
a(\boldsymbol{r}, t)=\int_{\omega-\Delta \omega}^{\omega+\Delta \omega} \int_{k-\Delta k}^{k+\Delta k} A\left(\boldsymbol{k}^{\prime}, \omega^{\prime}\right) \exp \left[i\left(\omega^{\prime} t-\boldsymbol{k}^{\prime} \cdot \boldsymbol{r}\right)\right] \mathrm{d} \boldsymbol{k}^{\prime} \mathrm{d} \omega^{\prime} .
$$

Integrating for the variable of $\boldsymbol{k}^{\prime}$, the solution of the dispersion relation for a given $\omega$ is taken as

$$
\boldsymbol{k}^{\prime}=\boldsymbol{k}^{\prime}(\omega)
$$

We take $k^{\prime}$ in the form such as Eq. (1),

$$
\boldsymbol{k}^{\prime}=k \boldsymbol{M}=k(\boldsymbol{\mu}-i \boldsymbol{\chi}),
$$

where $k$ is the wave number in vacuum which depends on wave frequency. The canonical equation for a ray path states that the direction of the ray is parallel to the normal to the refractive index surface and that the generalized Snell's law is to be satisfied. As to the former statement, Hines (1951) has shown that the direction of the ray is connected with only the real part of the complex refractive index vector by finding the maximum of the absolute amplitude of the wave packet with respect to the time and horizontal space coordinates, i.e., $\partial / \partial t=$ $\partial / \partial x=\partial / \partial y=0$ in the same coordinate system. This fact is in accordance with the condition that the exponent in Eq. (2), except the term of $[-k \chi \cdot r]$, must be stationary with respect to variations of the direction of $\mu$ and the wave number $k$ (the principle of stationary phase). Generalized Snell's law is also easily extended to the case of the absorbing medium by taking the real part of the refractive index vector. By adopting the definition of the ray direction and the generalized Snell's law, which are mentioned above, we can use the same canonical equation as given in the reference (BUDDEN, 1961), except that we should take the value of $\mu=|\mu|$ given in the reference for the value of $\mu=|\mu|$ from Eq. (4).

A set of differential equations for ray tracing is given as follows:

$$
\begin{aligned}
& \mathrm{d} x / \mathrm{d} t=\left(c / \mu^{2}\right)(\mu \sin \theta-\cos \theta \partial \mu / \partial \theta), \\
& \mathrm{d} z / \mathrm{d} t=\left(c / \mu^{2}\right)(\mu \cos \theta+\sin \theta \partial \mu / \partial \theta), \\
& \mathrm{d} \theta / \mathrm{d} t=-\left(c / \mu^{2}\right) \sin \theta \partial \mu / \partial z,
\end{aligned}
$$

in the two dimension. The quantities, $\mu$ and its derivatives, which are necessary for the righthand sides of Eq. (5) are obtained from the following simultaneous equations:

$$
\mu^{2}-\chi^{2}-2 i \mu \chi \cos \theta=M^{2}(\phi),
$$




$$
\begin{aligned}
& M^{2}(\phi)=1-2 X(1-X-i Z) / D, \\
& D=2(1-X-i Z)(1-i Z)-Y^{2} \sin ^{2} \phi+S, \\
& S= \pm \sqrt{Y^{4} \sin ^{4} \phi+4 Y^{2}(1-X-i Z)^{2} \cos ^{2} \phi}, \\
& \cos \phi=\{\mu \cos (\theta-\phi)-i \chi \cos \phi\} / M, \\
& \frac{\partial \mu}{\partial \theta}=\frac{E_{\mathrm{r}} C_{\mathrm{i}}-E_{\mathrm{i}} C_{\mathrm{r}}}{B_{\mathrm{r}} C_{\mathrm{i}}-B_{\mathrm{i}} C_{\mathrm{r}}}, \\
& \frac{\partial \mu}{\partial z}=\frac{F_{\mathrm{r}} C_{\mathrm{i}}-F_{\mathrm{i}} C_{\mathrm{r}}}{B_{\mathrm{r}} C_{\mathrm{i}}-B_{\mathrm{i}} C_{\mathrm{r}}}, \\
& B \equiv B_{\mathrm{r}}+i B_{\mathrm{i}}=A(\mu-i \chi \cos \theta)-M_{\frac{\partial M}{\partial \phi}} \cos (\theta-\phi), \\
& C \equiv C_{\mathrm{r}}+i C_{\mathrm{i}}=-A(\chi+i \mu \cos \theta)+i M \frac{\partial M}{\partial \phi} \cos \phi, \\
& E \equiv E_{\mathrm{r}}+i E_{\mathrm{i}}=-\mu\left\{A(i \chi \sin \theta)+M \frac{\partial M}{\partial \phi} \sin (\theta-\phi)\right\}, \\
& F \equiv F_{\mathrm{r}}+i F_{\mathrm{i}}=-M^{2} \sin \phi\left(\frac{\partial M}{\partial X} \frac{\partial X}{\partial z}+\frac{\partial M}{\partial Z} \frac{\partial Z}{\partial z}\right), \\
& A \equiv A_{\mathrm{r}}+i A_{\mathrm{i}}=\frac{\partial M}{\partial \phi} \cos \phi-M \sin \phi, \\
& \frac{\partial M}{\partial \phi}=\frac{\left(M^{2}-1\right) Y^{2} \sin \phi \cos \psi}{M D}\left[1-\frac{1}{S}\left\{Y^{2} \sin ^{2} \phi-2(1-X-i Z)^{2}\right\}\right], \\
& \frac{\partial M}{\partial X}=\frac{1}{M D}\left[2 X-1+i Z+\left(M^{2}-1\right)\left\{1-i Z+\frac{2}{S} Y^{2}(1-X-i Z) \cos ^{2} \phi\right\}\right], \\
& \frac{\partial M}{\partial Z}=\frac{i}{M D}\left[X+\left(M^{2}-1\right)\left\{2-X-2 i Z+\frac{2}{S} Y^{2}(1-X-i Z) \cos ^{2} \phi\right\}\right],
\end{aligned}
$$

where

$X=$ normalized electron density $=\left(N e^{2}\right) /\left(\omega^{2} m \varepsilon_{0}\right)$,

$Y=$ magnitude of the normalized earth's magnetic field vector $\boldsymbol{Y}=(e \boldsymbol{B}) /(\omega m)$,

$Z=$ normalized collision frequency $=\nu / \omega$,

$\psi=$ angle between $\boldsymbol{M}$ and $\boldsymbol{Y}$,

$\phi=$ angle between $z$-axis and $\boldsymbol{Y}$,

$N=$ electron density,

$e=$ charge on an electron,

$m=$ mass of an electron,

$\omega=$ angular wave frequency $=2 \pi f$,

$\varepsilon_{0}=$ dielectric constant of free space,

$\nu=$ collision frequency in collisions per second. 
The following equation is obtained by eliminating the variables $M$ and $\psi$ from Eqs. (6)-(10), from which we can derive the values, $\mu$ and $\chi$ as the functions of $\theta$.

where

$$
\alpha G^{2}+\beta G H+\gamma G+\delta=0,
$$

$$
\begin{aligned}
\alpha & =(1-i Z)^{2}(1-i Z-X)-(1-i Z) Y^{2}, \\
\beta & =X Y^{2}, \\
\gamma & =-X Y^{2}+2(1-i Z)(1-i Z-X) X, \\
\delta & =(1-i Z-X) X^{2}, \\
G & =\mu^{2}-\chi^{2}-1-2 i \mu \chi \cos \theta, \\
H & =[\mu \cos (\theta-\phi)-i \chi \cos \phi]^{2} .
\end{aligned}
$$

In our model, the direction of the local stratification of an electron density profile at the lower boundary of the ionosphere is assumed to be constant ( $x$ direction), or the vector $\chi$ is taken to have a constant direction ( $z$ direction). The vector $\boldsymbol{Y}$ is taken to be locally constant. Eqs. (5)-(20) can be applied to the ray paths only in the plane of the magnetic meridian and the refractive index vector $\boldsymbol{M}$ in a form like Eq. (1) has a real $x$ component, which is only applied to the case of the wave propagation being coupled with the electromagnetic waves in free space.

\section{Results of Numerical Calculation}

In the following calculation, we have used the representative physical parameters for the ionosphere at the altitude of several ten kilometers above the ground as follows; the electron density and the collision profile are given with respect to the altitude in Fig. 2: For $f=5 \mathrm{kHz}$, at $70 \mathrm{~km}$ height, $X=675.8$ and $Z=485.9$. At $80 \mathrm{~km}$ height, $X=3121.9$ and $Z=69.4$. At $90 \mathrm{~km}$ height, $X=$ 6432.0 and $z=9.7$.

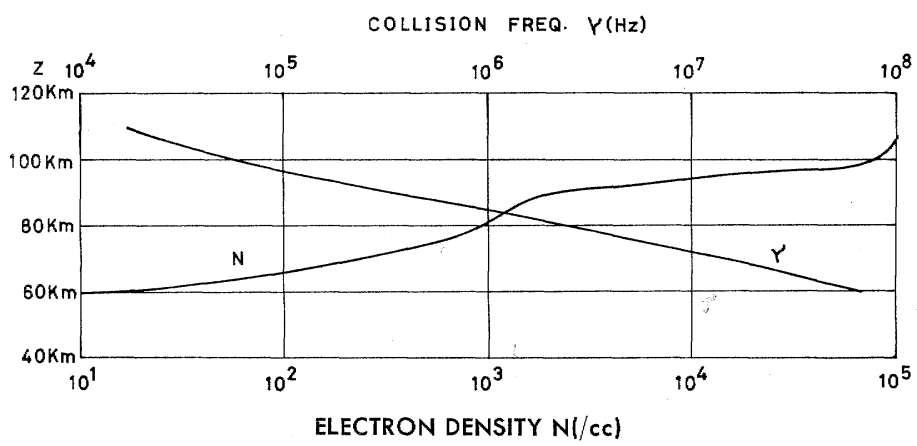

Fig. 2. Electron density $N$, and collision $\nu$, profile with respect to the altitude. 


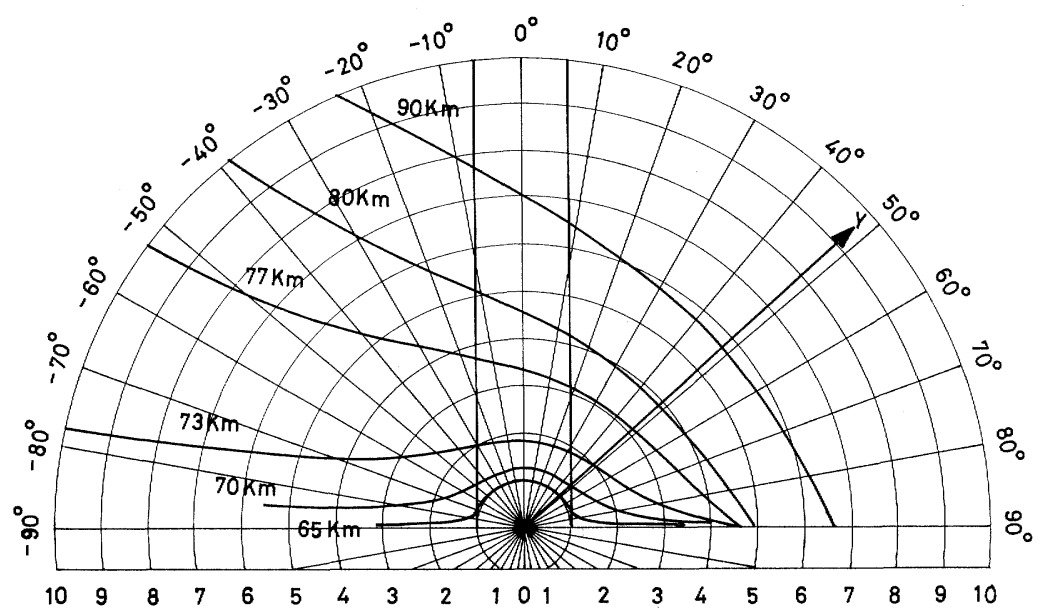

Fig. 3. Wave normal surfaces. Magnetic dip angle $\phi$ (the direction of $\boldsymbol{Y}$ ) is $48^{\circ}$. The values of $\mu$ as the function of $\theta$ are shown for $65,70,73,77,80$ and $90 \mathrm{~km}$ altitude, and the wave frequency $f$ is $5 \mathrm{kHz} . \quad Y=209.0$.

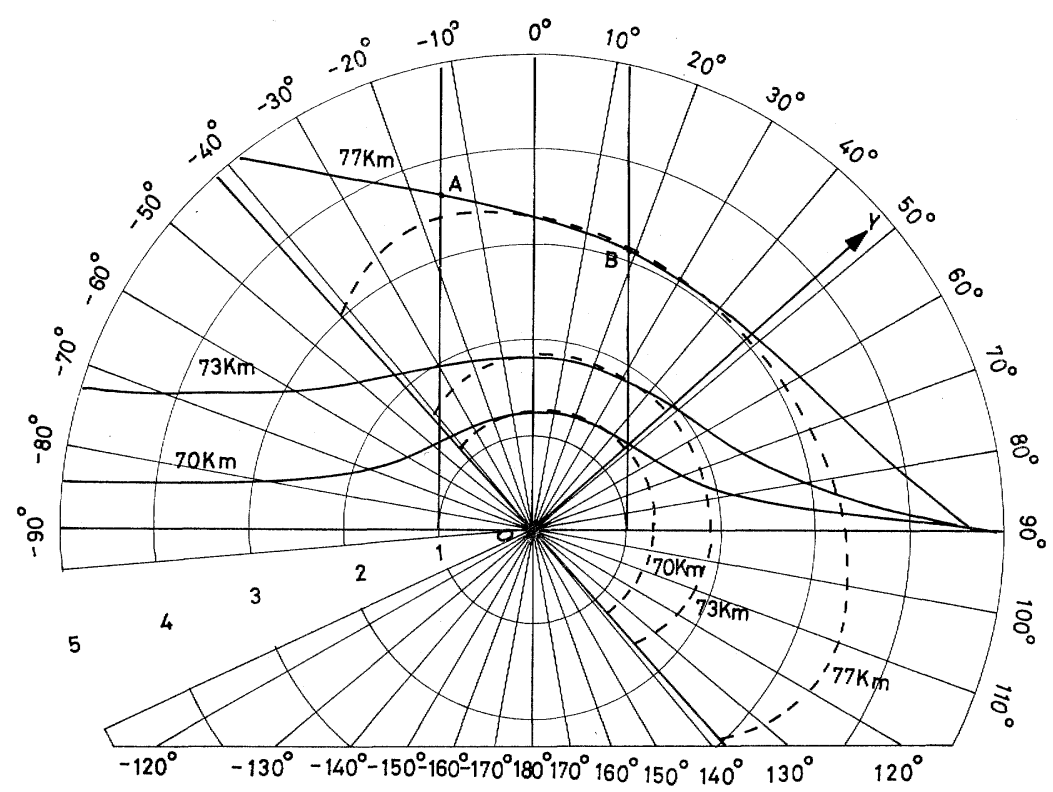

Fig. 4. In order to compare our solutions with the usual solutions of Appleton's formula, the values of $\mu$ as the function of $\theta$ are shown. Our solutions are denoted as solid lines and the usual solutions are denoted as dashed lines for the same parameters. Parameters used here are the same as those in Fig. 3.

Solutions of the dispersion relation, Eq. (21) are shown in Fig. 3, where we represent the values of $\mu$ as the function of $\theta$. Here, magnetic dip angle, $\phi$ is assumed to be $48^{\circ}$, which corresponds to our Kasugai Observatory $\left(24^{\circ}\right.$ in 
geomagnetic latitude). $Y=209.0$. The curves ( $\mu$ surfaces) above $90 \mathrm{~km}$ height are not shown in the figure, but they are nearly parallel to the curve for $90 \mathrm{~km}$ height. Considering that the direction of ray is expressed as the normal to these $\mu$ surfaces at each point, the ray path becomes the straight line above $90 \mathrm{~km}$ height in the range of our scope, which is to be shown later in Fig. 5.

In order to compare our solutions for $\phi=48^{\circ}$ with the usual solutions of Appleton's formula with real angle $\psi$, Fig. 4 is added, where solid lines correspond to our solutions and dashed lines correspond to usual solutions. In Fig. 4, for example, the angle $\angle A O B$ is the critical angle for the penetration of downcoming waves at $77 \mathrm{~km}$ height. The usual solutions are, of course, symmetric with respect to the direction of the magnetic line of force, while the characteristics of our solutions are affected by both directions of the magnetic dip and the normal to the stratification of electron density ( $\chi$-vector in Fig. 1). Two solutions do not seem to be so different. However, when the directions of wave normals part from that of the magnetic field, the directions of the normals to both curves (solid line and dashed line) separate by a certain amount, which leads to the differences of the ray paths of homogeneous waves and inhomogeneous waves.

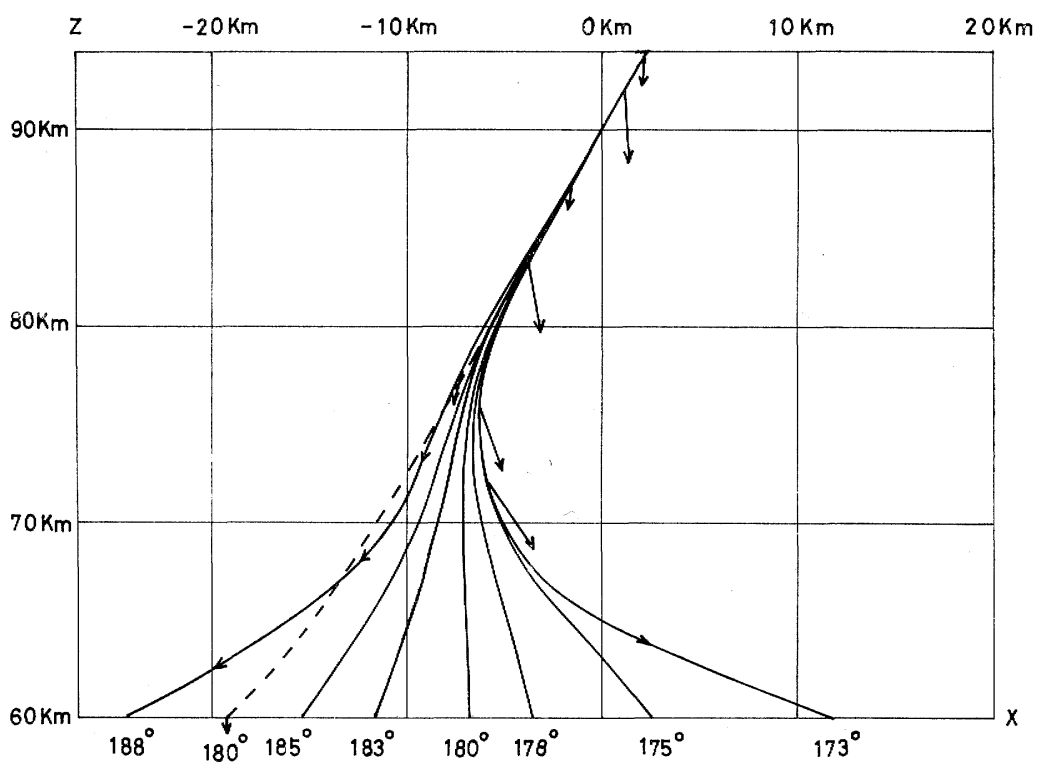

Fig. 5. Ray paths for the case of $\phi=48^{\circ}$, where other parameters used here are the same as those in Fig. 3. In order to elucidate the difference of the ray paths with various initial conditions, we made all ray paths accord at a point of $90 \mathrm{~km}$ height. The parameters $173^{\circ}, 175^{\circ}, 178^{\circ}, 180^{\circ}, 183^{\circ}$ and $185^{\circ}$ denote the polar angles of wave normal vectors at this point. Long arrows and short arrows express the directions of wave normals at each point for the case of $173^{\circ}$ and $188^{\circ}$, respectively. $Y=$ 209.0. The ray path for the collisionless case is denoted as a dashed line, where the initial condition of $\theta$ is $180^{\circ}$. 


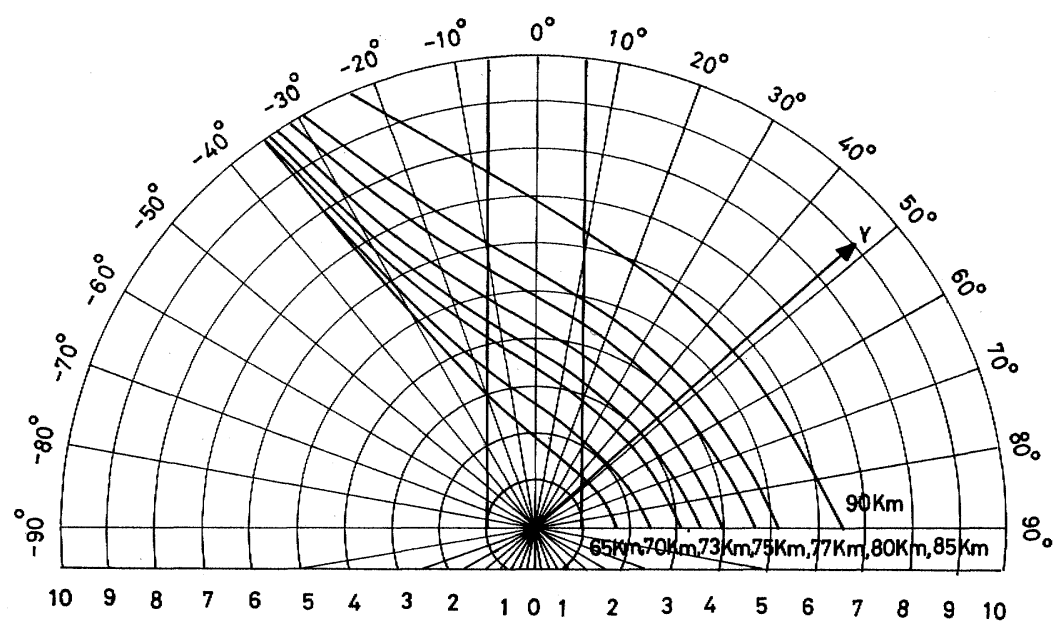

Fig. 6. Wave normal surfaces for collisionless case. All parameters are quite the same as those in Fig. 3, except that $\boldsymbol{Z}=\mathbf{0}$.

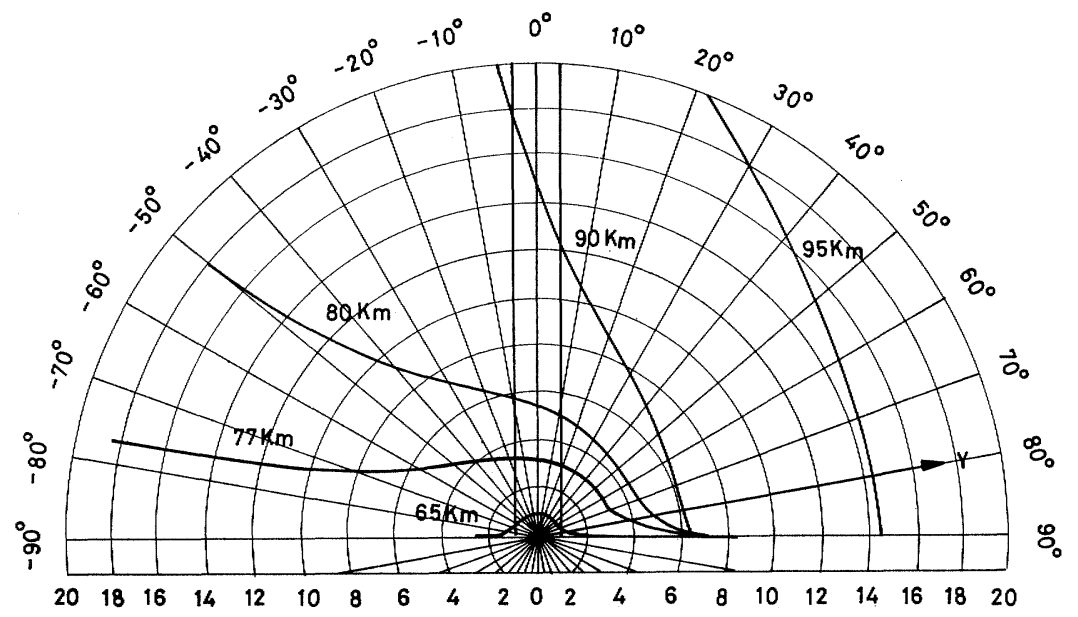

Fig. 7. Wave normal surfaces corresponding to the case of $\phi=80^{\circ}$. The values of $\mu$ as the function of $\theta$ are shown for $65,77,80,90$ and $95 \mathrm{~km}$ altitude, and the wave frequency $f$ is $5 \mathrm{kHz} . \quad Y=172.4$.

The computation of whistler ray paths were made by using Eq. (5) for the same parameters as in Fig. 3. The results are shown in Fig. 5. The parameters written under the $x$ axis in the figure denote the polar angles of wave normal vectors at the altitude of $90 \mathrm{~km}$. In order to elucidate the difference of the ray paths with various initial conditions, we made all ray paths accord at a point of $90 \mathrm{~km}$ height. The parameters mentioned above denote the directions of various wave normals within the critical angle at this point. Long arrows and short arrows show the directions of wave normals for the cases of both edges, i.e., the case of $173^{\circ}$ and $188^{\circ}$, respectively. If we neglect the collisional effects, 


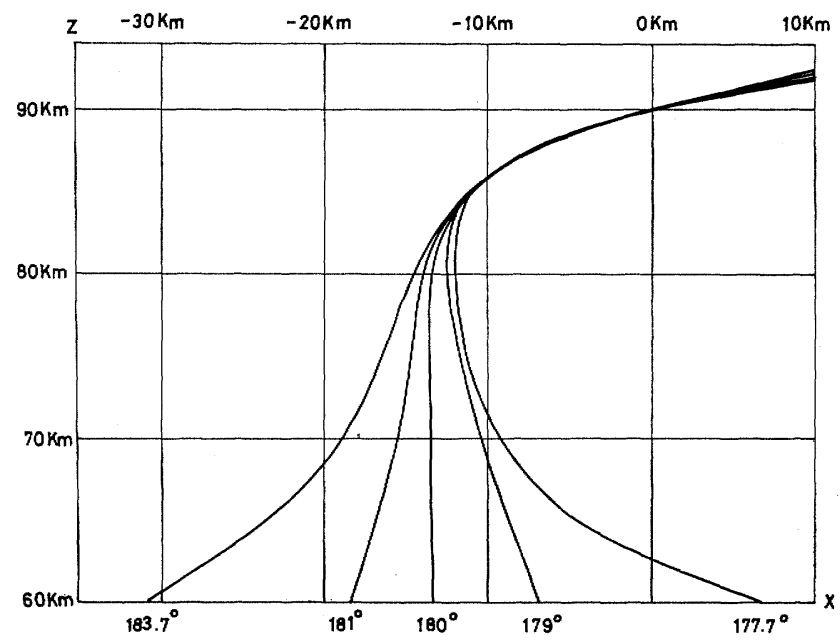

Fig. 8. Ray paths for the case of $\phi=80^{\circ}$. The parameters, $177.7^{\circ}, 179^{\circ}$, $180^{\circ}, 181^{\circ}$ and $183.7^{\circ}$, denote the polar angles of wave normal vectors at a point of $90 \mathrm{~km}$ height, which correspond to various initial conditions. The wave frequency $f$ is $5 \mathrm{kHz} . \quad Y=172.4$.

i.e., $Z=0$ in the case where other parameters are quite the same, the ray path is quite different. It is added in Fig. 5 for comparison as a dashed line for the initial condition of $\theta=180^{\circ}$. The wave normal surfaces in the collisionless case, $Z=0$, are shown in Fig. 6 , they run nearly parallel with one another within the critical angle. The value of the refractive index, $\mu$, remains to be large as compared with the value in the free space, unity, at the bottom of the ionosphere with our density profile, i.e., the gap of the value of the refractive index between the ionosphere and the free space becomes larger than that in the case of considering collisional effects. Consequently, the ray path becomes a nearly straight line in the ionosphere and shows a steep refraction at the boundary.

In Figs. 7 and 8, wave normal surfaces and ray paths for $\phi=80^{\circ}$ are presented to show the differences by the change of magnetic dip. The ray directions are stretched toward the direction of magnetic field. We can see the same trend for the case of other magnetic dips, which are not shown here.

\section{Conclusion and Discussion}

(1) This subject has a starting point in the description in Helliwell's textbook (1965), concerning the contribution of collisional effects to the coupling from electromagnetic waves in free space to whistler modes in the ionosphere. We have found how the downcoming whistler wave whose wave normal lies within the critical angle penetrates free space, while the downcoming wave whose wave normal lies outside of the critical angle will reflect perfectly near 
the bottom of the ionosphere. As shown in the legend for Fig. 5, the collisional effects make the refraction of the ray path through the bottom of the ionosphere smooth, i.e., the variation of the refractive index from the ionosphere to the free space becomes continuous by the collisional effects.

(2) The justification of the application of ray theory to such a narrow region may be required. It is significant in the methodology to make the straight extension of usual ray equations, for the purpose of considering the collisional effects. However, ray tracing for such a low frequency wave in this narrow region is not a good example of the application of the present method. Although we can not justify our approximation clearly without showing the results of the full wave theory on the same problem, we should show the results of the ray tracing of higher frequency waves, for which the ray theory is appropriate. In Fig. 9, the ray paths with the initial condition of $\theta=180^{\circ}$ for higher frequency waves are shown to make a comparison to the case of $f=5 \mathrm{kHz}$. The ray path changes continuously with an increase in frequency. In Fig. 10, we show the ratio of the variation of the refractive index, $\mu$, per one wavelength to the refractive index itself, $(1 / 2 \pi)\left(\lambda_{0} / \mu^{2}\right) \partial \mu / \partial z$, for the cases of ray paths shown in Fig. 9 , where $\lambda_{0}$ is the wavelength in free space. If this value is sufficiently small as compared with unity, the ray tracing may be allowable by physical consideration. According to Fig. 10, the ray tracing will be permissible for the case of frequency higher than $100 \mathrm{kHz}$ in the region under discussion, and for the cases

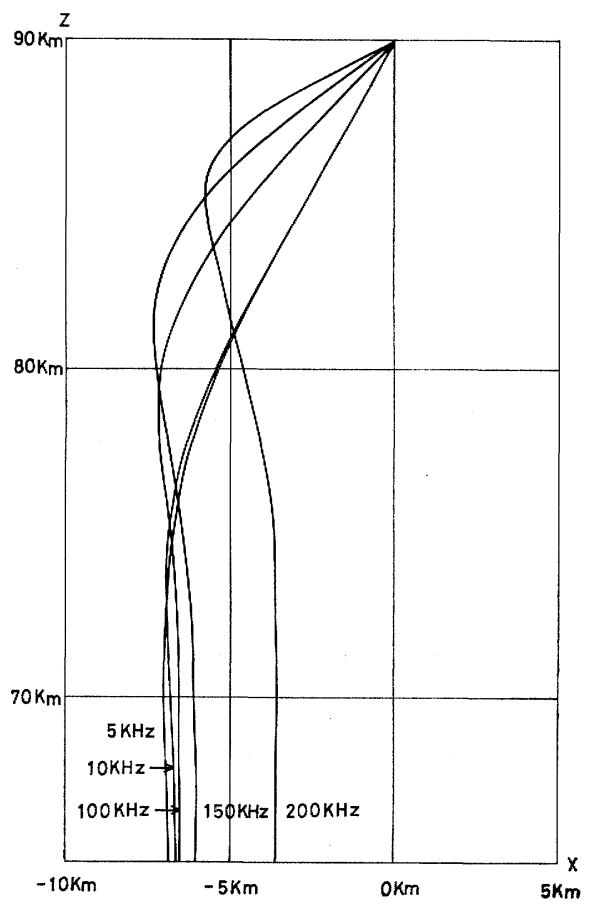

Fig. 9. Ray paths for the case of $\phi=48^{\circ}$. The initial condition of the direction of wave normal, $\theta$ is $180^{\circ}$ for all ray paths. The wave frequencies are taken to be $5,10,100,150$ and $250 \mathrm{kHz}$. 


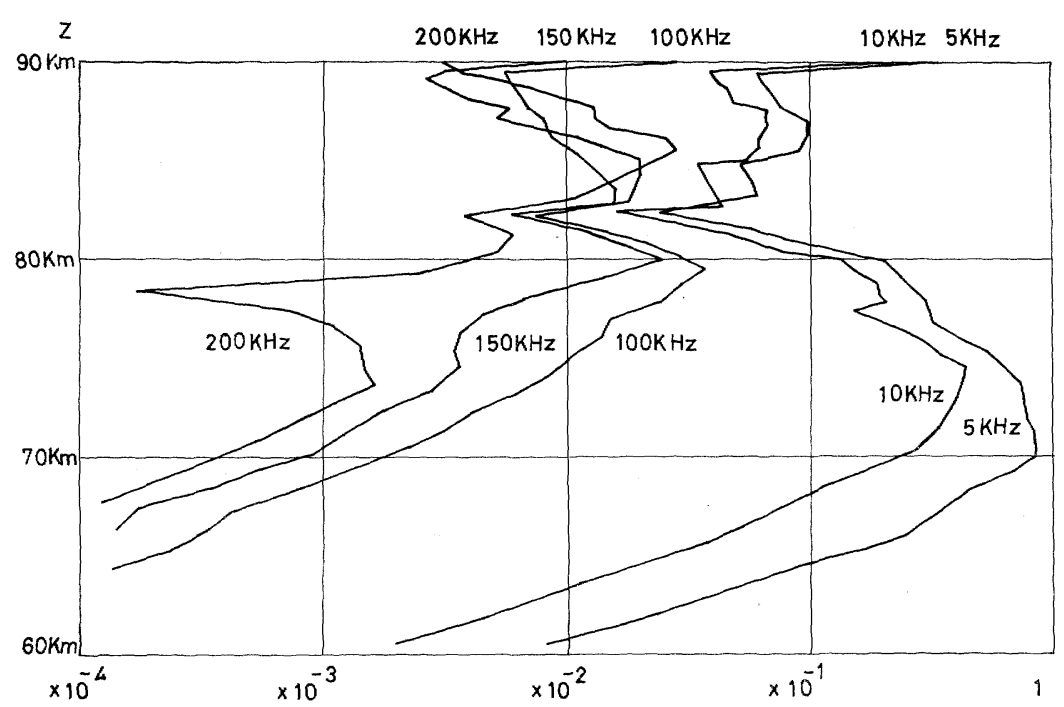

Fig. 10 The value of $(1 / 2 \pi)\left(\lambda_{0} / \mu^{2}\right) \mathrm{d} \mu / \mathrm{d} z$ is shown to investigate the justification of ray theory. Parameters are the same as in Fig. 9.

of $10 \mathrm{kHz}$ and $5 \mathrm{kHz}$, the condition of ray theory is not satisfied partly.

(3) Our formulation has the restriction described in Section 2. It cannot be applied to the tracing of total ray paths in the curved ionosphere. For such a case, the dispersion relation should be solved by considering that the projection of the refractive index vector $\boldsymbol{M}=\boldsymbol{\mu}-i \chi$ on the direction of each local stratification of electron density is to be conserved through all the steps of propagation (Snell's law), and Eq. (5) should be also modified. In our case, the $x$ component of the vector $\mu$ is automatically conserved by Eq. (5) and the $x$ component of vector $\chi$ is constant (zero).

(4) We have made the calculation of ray paths expressed in terms of the propagation of wave packet. The difference between the procedure of packet method and the procedure based on the direction of the Poynting vector should be discussed. Kelso (1964) introduced the following fact in his textbook, which was found and discussed by ScotT (1950) and Hines (1951): "In the case of a dispersive and anisotropic, but nondissipative and homogeneous medium, the same ray direction is obtained by both methods, but in a dissipative, horizontally stratified medium, the Poynting-vector method yields a westward flow of energy for vertical propagation, whereas the packet does not show this westward flow." We dealt with this problem as follows: to begin with, we ascertained Kelso's description by numerical calculation for our case and next, we did not adopt the Poynting-vector method by the reason mentioned afterward. In this method, we use the Poynting-vector as follows,

$$
\boldsymbol{S}=\operatorname{Re}\left(\boldsymbol{E} \times \boldsymbol{H}^{*}\right),
$$




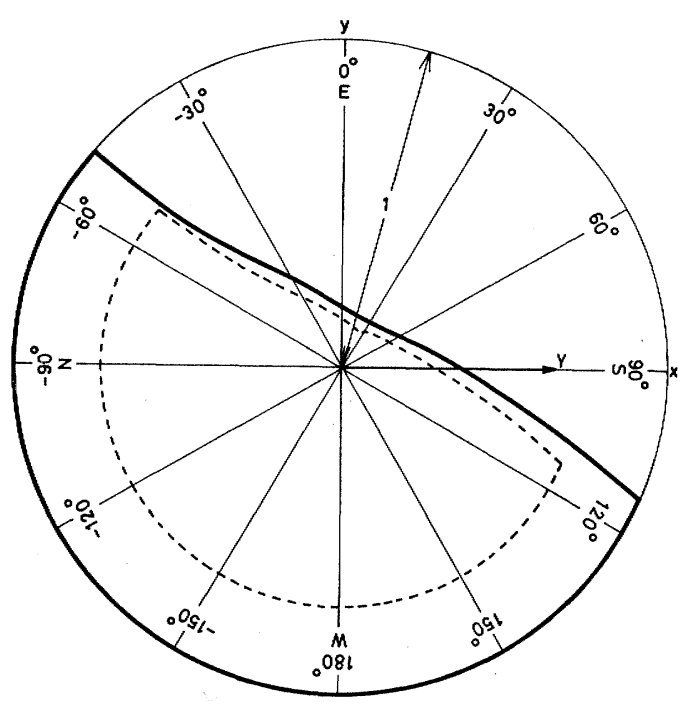

Fig. 11. Direction of wave normal vector $\mu$ which accompanies the westward flow of energy by the Poynting-vector method. The intersection of the direction of $\mu$ which satisfies the condition, $S_{y}<0$ and the unit spherical surface comes into the inside of a solid line for the case of $60 \mathrm{~km}$ height and into the inside of a dashed line for the case of $70 \mathrm{~km}$ height.

where the symbol * denotes complex conjugate, and we use the usual boundary conditions at the sharp boundary (WAIT, 1970). We calculate the direction of $\boldsymbol{S}$ for a given direction of $\mu, \theta$. In Fig. 11 we show the direction of real wave normal $\mu$ for which the Poynting-vector turns westward, i.e., $S_{y}<0$ (the direction of positive $y$ expresses the eastward). In Fig. 11, the intersection of the direction of $\mu$ which satisfies the condition, $S_{y}<0$ and the unit spherical surface comes into the inside of the solid line for the case of $60 \mathrm{~km}$ height (just at the bottom of the ionosphere) and into the inside of the dashed line for the case of $70 \mathrm{~km}$ height. The calculation is confined to the case of the upward wave normal vector within the cone of the critical angle, where $\phi=48^{\circ}, f=5 \mathrm{kHz}$ and other parameters are the same as those in the preceding section. This result involves the fact that the Poynting-vector method yields a westward flow of energy for vertical propagation. By considering the wave normal surface in three dimensions, the packet method is found to show the symmetry of the ray direction with respect to the direction of magnetic field. It never occurs in the packet method that the eastward $\mu$ accompanies the westward energy flow, which is natural from the physical viewpoint and is one of the reasons for adopting the packet method. Furthermore, the concept of the Poynting-vector to be interpreted as energy flow was first derived in the following integral form by the Poynting theorem as, 


$$
\int_{s_{0}}(\boldsymbol{E} \times \boldsymbol{H}) \cdot \mathrm{d} \boldsymbol{S}_{0}
$$

It is of course correct to interpret this integral as the flux of energy in the electromagnetic field per second crossing the area $\boldsymbol{S}_{0}$, while the integrand, $\boldsymbol{E} \times \boldsymbol{H}$ cannot always be interpreted as the intensity of energy flow because of the arbitrariness of the terms which vanish through the surface integral in Eq. (23).

(5) The effect of ions on the ray tracing have been neglected in our calculation. Kimura (1966) reminds us that the frequency limit below which the effect of ions becomes important on ray tracing can be roughly determined by the lower hybrid resonance frequency and that it becomes $8 \mathrm{kHz}$, which of course depends on the physical parameters as the constituent of positive ions and the geomagnetic latitude besides the parameter of the electron density profile. The ray tracing of whistler modes with the frequency of $5 \mathrm{kHz}$ without considering the effect of ions have been insufficient in the actual condition. This problem is interesting but is outside the present theme.

We wish to express our sincere thanks to Professor A. Kimpara for continual guidance. We are very grateful to Dr. Y. Midzuno at Institute of Plasma Physics, Nagoya University for stimulating discussions. We are indebted to Dr. Takashi Araki at Geophysical Institute, Tohoku University for offering density and collision profiles, and for valuable discussions. Our thanks for tracing the figures are due to Miss M. Kinoshita.

\section{REFERENCES}

Budden, K.G., Radiowaves in the Ionosphere, pp. 235 and 237, Cambridge Univ. Press, London, 1961.

Haselgrove, J., Ray theory and new method for ray tracing, Proc. Camb. Conf. Phys. Ionosphere, 355-364, Phys. Soc. London, 1955.

Helliwell, R.A., Whistlers and Related Ionospheric Phenomena, pp. 27, Stanford Univ. Press, Stanford, 1965.

Hines, C.O., Wave packets, the Poynting-vector, and energy flow. Part III, J. Geophys. Res., 56, 207-220, 1951.

Kelso, J.M., Radio Ray Propagation in the Ionosphere, pp. 344-347, McGraw-Hill, New York, 1964.

Kimura, I., Effects of ions on whistler-mode ray tracing, Radio Sci., 1, 269-283, 1966.

MAEDA, K. and H. OYA, Penetration of VLF radio waves through the ionosphere, J. Geomag. Geoelectr., 14, 151-171, 1963.

Scott, J.C.W., The Poynting-vector in the ionosphere, Proc. I.R.E., 38, 1057-1068, 1950.

WaIt, J.R., Electomagnetic Waves in Stratified Media, pp. 239-243, Pergamon Press, Oxford, 1970.

YABROFF, I., Computation of whistler ray paths, J. Res. NBS., 65D, 485-505, 1961. 